\title{
Design, Optimization, and Characterization of Lactoferrin-loaded Chitosan/TPP and Chitosan/Sulfobutylether- $\beta$-cyclodextrin Nanoparticles as a Pharmacological Alternative for Keratoconus Treatment
}

Rubén Varela-Fernández ${ }^{1,2}$, Xurxo García-Otero ${ }^{1,3}$, Victoria Díaz-Tomé ${ }^{1}$, Uxía Regueiro², Maite LópezLópez ${ }^{2}$, Miguel González-Barcia ${ }^{4}$, María Isabel Lema ${ }^{5,}$ and Francisco Javier Otero-Espinar ${ }^{1,6, *}$.

1. Department of Pharmacology, Pharmacy and Pharmaceutical Technology. University of Santiago de Compostela (USC). Campus vida. Santiago de Compostela. Zip Code: 15782. Spain. francisco.otero@usc.es.

2. Clinical Neurosciences Group. University Clinical Hospital, Health Research Institute of Santiago de Compostela (IDIS). Travesía da Choupana s/n Santiago de Compostela. Zip Code: 15706. Spain. rubenvf1@gmail.com.

3. Molecular Imaging Group. University Clinical Hospital, Health Research Institute of Santiago de Compostela (IDIS).

Travesía da Choupana s/n Santiago de Compostela. Zip Code: 15706. Spain. xurxo.garcia.otero@gmail.com

4. Clinical Pharmacology Group. University Clinical Hospital, Health Research Institute of Santiago de Compostela (IDIS).

Travesía da Choupana s/n Santiago de Compostela. Zip Code: 15706. Spain. miguel.gonzalez.barcia@sergas.es.

5. Department of Surgery and Medical-Surgical Specialties. Ophthalmology Area. University of Santiago de Compostela (USC).Campus Vida. Santiago de Compostela. Zip Code: 15706. Spain. mariaisabel.lema@usc.es.

6. Paraquasil Group. University Clinical Hospital, Health Research Institute of Santiago de Compostela (IDIS). Travesía da Choupana s/n Santiago de Compostela. Zip Code: 15706. Spain. francisco.otero@usc.es.

\section{EXPERIMENTAL METHODS}

Mucoadhesion study (page 6)

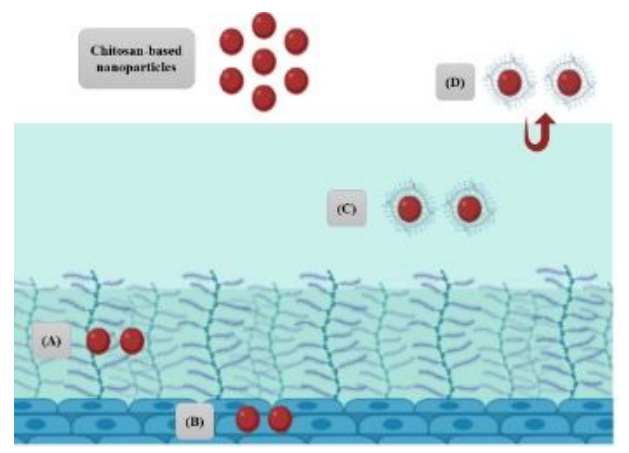

Figure S-1. Different approaches of the interaction between chitosan-based nanoparticles and the ocular mucin layer. (A) adsorption of the nanoparticles to the mucus layer, (B) passage through the mucin layer and reach the cornea, (C) loss of functionality of the nanoparticles when covered by mucin, or (D) nanoparticle's removal with the mucus layer renewal.

\section{RESULTS AND DISCUSSION}

Screening study: CS/TPP and CS/SBE- $\beta-C D$ nanoparticles formation.

Preparation of CS/TPP nanoparticles (page 8)
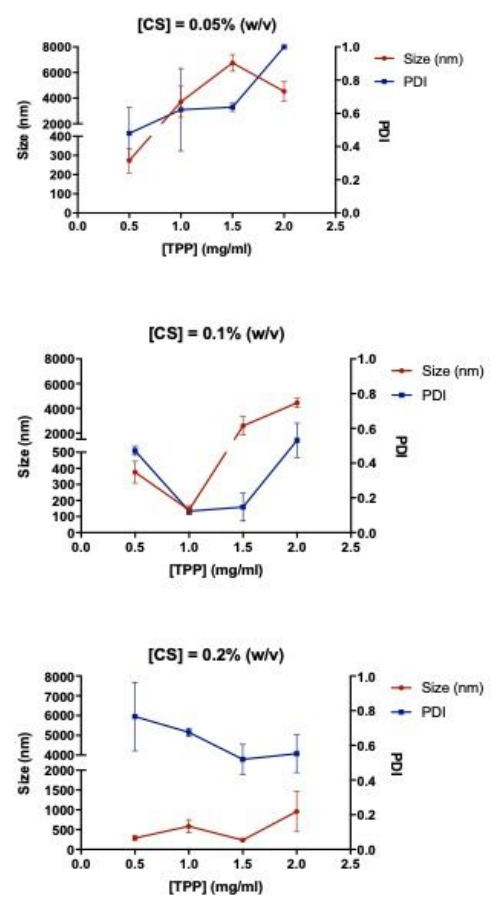
Figure S-2. Changes in size and PDI values during the optimization procedure of CS/TPP nanoparticles.

\section{Preparation of CS/SBE- $\beta$-CS nanoparticles (page 8)}
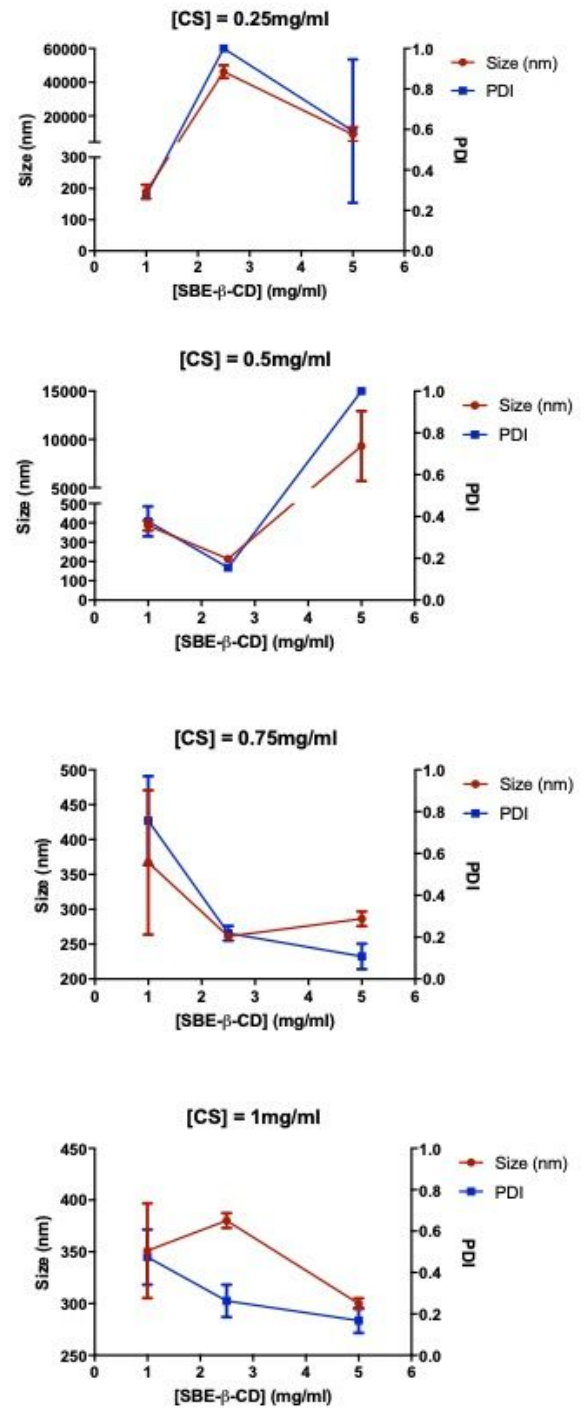

Figure S3. Changes in size and PDI values during the optimization procedure of $\mathrm{CS} / \mathrm{SBE}-\beta-\mathrm{CD}$ nanoparticles.

\section{Morphological evaluation (page 9)}
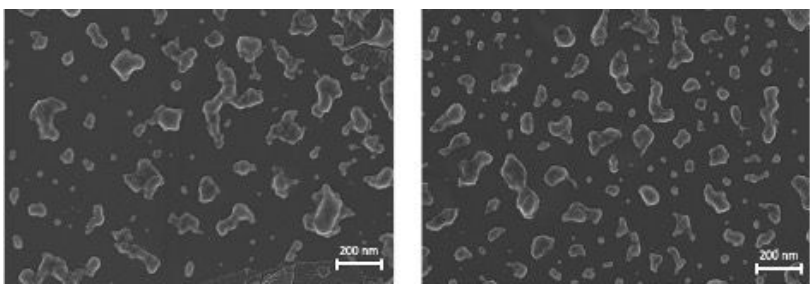

Figure S-4. SEM images of chitosan-based nanoparticles (CS/TPP NPs and CS/SBE-b-CD NPs, respectively).

\section{Stability studies.}

\section{Stability to storage (page 11)}
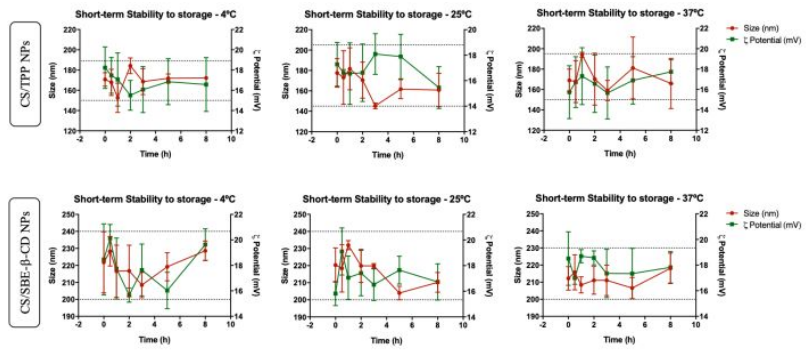

Figure S-5. Long-term stability-to-storage study for CS/TPP and CS/SBE- $\beta-C D$ NPs (up and down, respectively) over a 3-month period. Stripped lines show the $10 \%$ variation size interval from the initial size values, which comprises the nanoparticle stability range.

Cytotoxicity analysis.

Bovine Corneal Opacity and Permeability test (BCOP) (page 13)
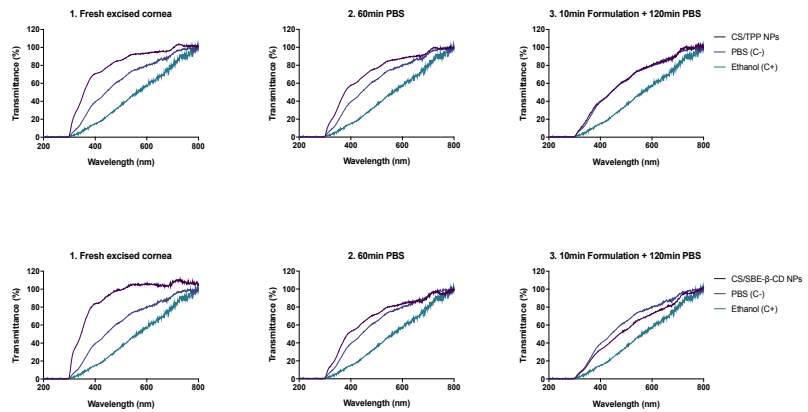

Figure S-6. BCOP results for chitosan-based nanoparticles. (I) Evolution of corneal transparency for CS/TPP nanoparti-cles over time during BCOP assay, (II) Evolution of corneal transparency for CS/SBE-b-CD nanoparticles over time during BCOP assay, and (III) Resulting data of corneal transparency, opalescence and fluorescein permeability for CS/TPP and CS/SBE-b-CD nanoparticles.

Hen's egg test on the chorioallantoic membrane (HET-CAM) (page 13)
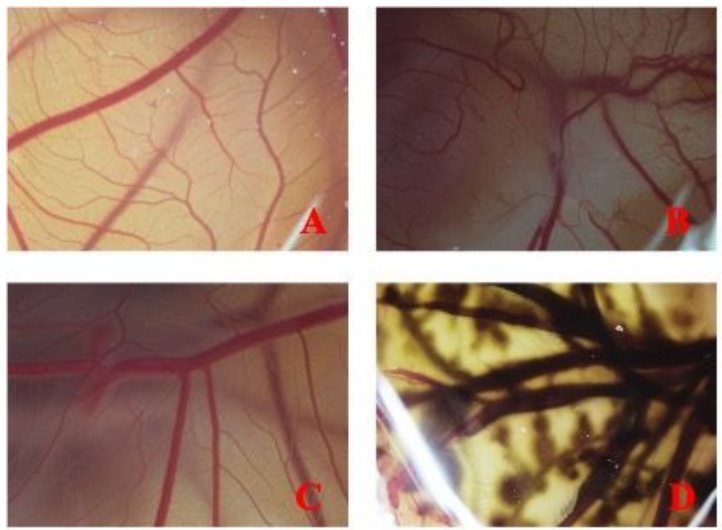
Figure S-7. Images of CAM membranes after the chitosan-based nanoparticles administration during the HET-CAM test, compared to the control solutions. (A) CS/TPP nanoparticles, (B) CS/SBE-b-CD nanoparticles, (C) BSS (negative control), and (D) $\mathrm{NaOH}$ (positive control).

In vivo ocular surface permanence study.

Evaluation of the radiolabeling stability and efficiency of chitosan-based nanoparticles (page 14)
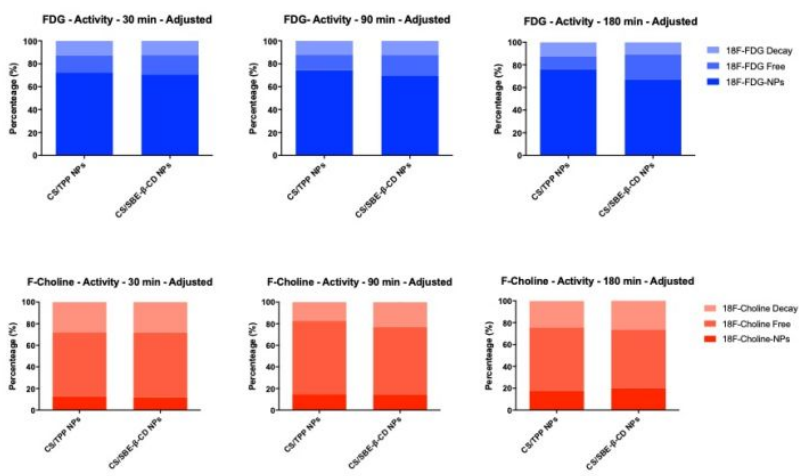

Figure S-8. Radiotracer labeling stability and efficiency for chitosan-based nanoparticles over time. (I) 18F-FDG (above), and (II) 18F-Choline (below). 